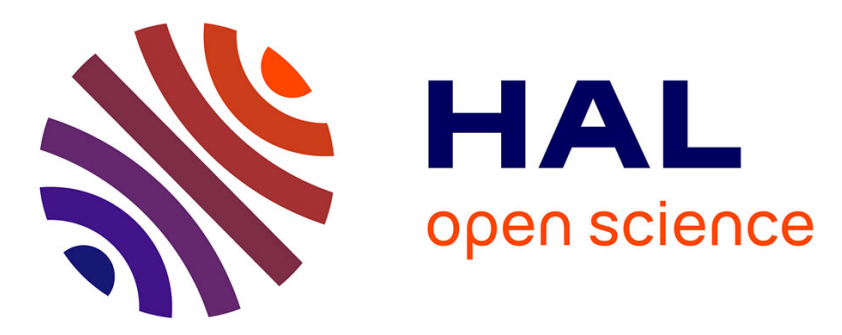

\title{
Monitor-Based Statistical Model Checking for Weighted Metric Temporal Logic
}

\author{
Peter Bulychev, Alexandre David, Kim Guldstrand Larsen, Axel Legay, \\ Guangyuan Li, Danny Bogsten Poulsen, Amélie Stainer
}

\section{To cite this version:}

Peter Bulychev, Alexandre David, Kim Guldstrand Larsen, Axel Legay, Guangyuan Li, et al.. Monitor-Based Statistical Model Checking for Weighted Metric Temporal Logic. Logic for Programming, Artificial Intelligence, and Reasoning, Mar 2012, Merida, Venezuela. pp.168-182. hal-00744100

\author{
HAL Id: hal-00744100 \\ https://hal.inria.fr/hal-00744100
}

Submitted on 22 Oct 2012

HAL is a multi-disciplinary open access archive for the deposit and dissemination of scientific research documents, whether they are published or not. The documents may come from teaching and research institutions in France or abroad, or from public or private research centers.
L'archive ouverte pluridisciplinaire HAL, est destinée au dépôt et à la diffusion de documents scientifiques de niveau recherche, publiés ou non, émanant des établissements d'enseignement et de recherche français ou étrangers, des laboratoires publics ou privés. 


\title{
Monitor-Based Statistical Model Checking for Weighted Metric Temporal Logic
}

\author{
Peter Bulychev ${ }^{1}$, Alexandre David ${ }^{1}$, Kim G. Larsen ${ }^{1}$, Axel Legay ${ }^{2}$, \\ Guangyuan $\mathrm{Li}^{3}$, Danny Bøgsted Poulsen ${ }^{1}$, Amelie Stainer ${ }^{2}$ \\ 1 Computer Science, Aalborg University, Denmark \\ 2 INRIA/IRISA, Rennes Cedex, France \\ 3 State Key Laboratory of Computer Science, Institute of Software, The Chinese \\ Academy of Sciences, Beijing, P.R. of China
}

\begin{abstract}
We present a novel approach and implementation for analysing weighted timed automata (WTA) with respect to the weighted metric temporal logic $\left(\mathrm{WMTL}_{\leq}\right)$. Based on a stochastic semantics of WTAs, we apply statistical model checking (SMC) to estimate and test probabilities of satisfaction with desired levels of confidence. Our approach consists in generation of deterministic monitors for formulas in $\mathrm{WMTL}_{\leq}$, allowing for efficient SMC by run-time evaluation of a given formula. By necessity, the deterministic observers are in general approximate (over- or under-approximations), but are most often exact and experimentally tight. The technique is implemented in the new tool CASAAL that we seamlessly connect to UPPAAL-SMC in a tool chain. We demonstrate the applicability of our technique and the efficiency of our implementation through a number of case-studies.
\end{abstract}

\section{Introduction}

Model checking (MC) [14] is a widely used approach to guarantee correctness of a system by checking that its model satisfies a given property. A typical model checking algorithm explores a state space of a model and tries to prove or disprove that the property holds on the model.

Despite a large and growing number of successful applications in industrial case studies, the MC approach still suffers from the so-called state explosion problem. This problem manifests itself in the form of unmanageably large state spaces of models with large number of components (i.e. number of variables, parallel components, etc). The situation is even worse when a system under analysis is hybrid (i.e. it possesses both continuous and discrete behaviors), because a state space of such models may lack finite representation [2]. Another challenge for $\mathrm{MC}$ is to analyze stochastic systems, i.e. systems with probabilistic assumptions for their behavior.

One of the ways to avoid these complexity and undecidability issues is to use statistical model checking (SMC) approach [19]. The main idea of the latter is to observe a number of simulations of a model and then use results from statistics (e.g. sequential analysis) to get an overall estimate of a system behavior. 
In the present paper we consider a problem of computing the probability that a random run of a given weighted timed automaton (WTA) satisfies a given weighted metric temporal logic formula $\left(\mathrm{WMTL}_{\leq}\right)$. Solving this problem is of great practical interest since WTA are as expressive as general linear hybrid automata [2], a formalism which has proved to be very useful for modeling real-world hybrid and real-time systems. Moreover, $\mathrm{WMTL}_{\leq}[7]$ is not only a weighted extension of the well established LTL but can also be seen as an extension of MTL [15] to hybrid systems. However, the model checking problem for $\mathrm{WMTL}_{\leq}$is known to be undecidable [7], and in our paper we propose an approximate approach that computes a confidence interval for the probability. In most of the cases this confidence interval can be made arbitrary small.
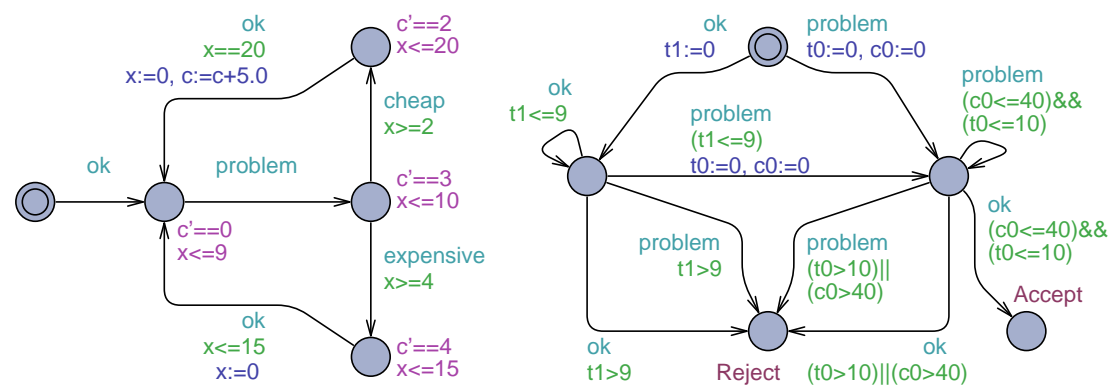

Fig. 1: A model (left) and deterministic monitor (right) for the repair problem

As an example consider a never-ending process of repairing problems [7], whose Weighted Timed Automata model are depicted at Fig. 1 (left). The repair of a problem has a certain cost, captured in the model by the clock $\mathrm{c}^{1}$. As soon as a problem occurs (modeled by the transition labeled by action problem) the value of c grows with rate 3, until actual cheap (rate 2) or expensive (rate 4) repair is taking place. Clock $\mathrm{x}$ grows with rate 1 (it's default behavior unless other rate is specified). Being a Weighted Timed Automata, this model is equipped with a natural stochastic semantics [10] with a uniform choice on possible discrete transitions and uniformly selected delays in locations.

Now consider that we want to express the property that a path goes from ok back to itself in time less than 10 time units and cost less than 40 . This can be formalized by the following $\mathrm{WMTL}_{\leq}$formula:

$$
\text { ok } \mathrm{U}_{\leq 9}^{\tau}\left(\text { problem } \wedge\left(\neg \mathrm{ok} \vee \mathrm{U}_{\leq 10}^{\tau} \text { ok }\right) \wedge\left(\neg \mathrm{ok} \mathrm{U}_{\leq 40}^{c} \mathrm{ok}\right)\right)
$$

Here, the MITL $\mathrm{M}_{\leq}$-formula $\varphi_{1} \mathrm{U}_{\leq d}^{c} \varphi_{2}$ is satisfied by a run if $\varphi_{1}$ is satisfied on the run until $\varphi_{2}$ is satisfied, and this will happen before the value of the clock $c$ increases with more than $d$ starting from the beginning of the run $(\tau$ is a special clock that always grows with rate 1 ).

${ }^{1}$ we will (mis) use the term "clock" from timed automata, though in the setting of WTAs the clocks are really general real-valued variables 
In order to estimate the probability that a random run of a model satisfies a given property, our approach will first construct deterministic monitoring weighted timed automata for this property. In fact, it is not always possible to construct an exact deterministic observer for a property ${ }^{2}$, thus our tool can result in deterministic under- and over-approximations. For our example, the tool constructed the exact deterministic monitor presented in Fig. 1 (right). Here rates of a monitoring automaton are defined by the rates of the automaton being monitored, i.e. the rate of $\mathrm{c} 0$ is equal to the rate of $\mathrm{c}$.

The constructed monitoring WTA permits the SMC engine of UPPAAL to use run-time evaluation of the property in order to efficiently estimate the probability that runs of the models satisfy the given property. In our example the UPPAALSMC returns the $95 \%$ confidence interval $[0.215,0.225]$. If none of the under- and over-approximation monitors are exact, then we use both of them to compute the confidence interval.

Our contribution is twofold. First, we are the first to extend statistical model checking to the $\mathrm{WMTL}_{\leq}$logic. The closest logic that has been studied so far is the strictly less expressive $\mathrm{MITL}_{\leq}$, that does not allow to use energy clocks in the $\mathrm{U}$ operator. Second, our monitor-based approach works on-the-fly and can terminate a simulation as soon as it may conclude that a formula will be satisfied (or violated) by the simulation. Other statistical model checking algorithms that deal with linear-time properties (cf. $[1,18,19,20])$ require a posterior (and expensive) check after a complete simulation of a fixed duration has been generated.

\section{Weighted Timed Automata \& Metric Temporal Logic}

In this section we describe weighted timed automata (WTA) and weighted metric temporal logic $\left(\mathrm{WMTL}_{\leq}\right)$as our modeling and specification formalisms. A notion of monitoring weighted timed automata (MWTA) is used to define automatically constructed (deterministic) observers for $\mathrm{WMTL}_{\leq}$properties.

\subsection{Weighted Timed Automata}

Let $C$ be a set of clocks. A clock bound over $C$ has the form $c \sim n$ where $c \in C$, $\sim \in\{<, \leq, \geq,>\}$. We denote the set of all possible clock bounds over $C$ by $\mathcal{B}(C)$. A valuation over $C$ is a function $v: C \rightarrow \mathbb{R}_{\geq 0}$, and a rate vector is a function $r: C \rightarrow \mathbb{Q}$. We let $\mathcal{V}(C)(\mathcal{R}(C)$, respectively) to be all clock valuations (rates) over $C$.

Definition 1. A Weighted Timed Automaton ${ }^{3}$ (WTA) over alphabet $\mathcal{A}$ is a tuple $\left(L, \ell_{0}, C_{i}, C_{o}, E, W, I, R\right)$ where:

\footnotetext{
${ }^{2}$ For instance, $\diamond_{\leq 1}^{\tau}\left(p \wedge \square_{\leq 1}^{\tau}(\neg r) \wedge \diamond_{\leq 1}^{\tau}(q)\right)$ is a $\mathrm{WMTL}_{\leq}$property which can not be determinized, as proved in Appendix A

${ }^{3}$ In the classical notion of priced timed automata [6,4] cost-variables (e.g. clocks where the rate may differ from 1) may not be referenced in guards, invariants or in resets, thus making e.g. optimal reachability decidable. This is in contrast to our notion of WTA, which is as expressive as linear hybrid systems [8].
} 
- $L$ is a finite set of locations,

$-\ell_{0} \in L$ is the initial location,

$-C_{i}$ and $C_{o}$ are finite set of real-valued variables called internal clocks and observable clocks, respectively,

- $E \subseteq L \times \mathcal{A} \times 2^{\mathcal{B}\left(C_{i} \cup C_{o}\right)} \times 2^{C_{i}} \times L$ is a finite set of edges,

- W: $E \rightarrow \mathcal{R}\left(C_{i} \cup C_{o}\right)$ assigns weights to edges, weights of observable clocks should be non-negative (i.e. $W(e)(c) \geq 0$ for any $e \in E$ and $c \in C_{o}$ ),

- $I: L \rightarrow 2^{\mathcal{B}\left(C_{i} \cup C_{o}\right)}$ assigns an invariant to each location,

$-R: L \rightarrow \mathcal{R}\left(C_{i} \cup C_{o}\right)$ assigns rates to the clocks in each location, rates of observable clocks should be non-negative.

If $\delta \in \mathbb{R}_{\geq 0}$, then we define $v+\delta$ to be equal to the valuation $v^{\prime}$ such, that for all $c \in C$ we have $v^{\prime}(c)=v(c)+\delta$. If $r$ is a rate vector, then $v+r \cdot \delta$ is the valuation $v^{\prime}$ such that for all clocks $c$ in $C, v^{\prime}(c)=v(c)+r(c) \cdot \delta$. The valuation that assigns zero to all clocks is denoted by $\overrightarrow{0}$. Given $Y \subseteq C, v[Y=0]$ is the valuation equal to $\overrightarrow{0}$ over $Y$ and equal to $v$ over $C \backslash Y$. We say, that a valuation $v$ satisfies a clock bound $b=c \sim n$ (denoted $v \vDash b$ ), iff $v(c) \sim n$. A valuation satisfies a set of clock bounds if it satisfies all of them or this set is empty. A state $(l, v)$ of a WTA consists of a location $l \in L$ and a valuation $v \in \mathcal{V}\left(C_{i} \cup C_{o}\right)$. In particular, the initial sate of the WTA is $\left(\ell_{0}, \overrightarrow{0}\right)$. From a state a WTA can either delay for some time $\delta$ or it can perform a discrete action $a$, the rules are given below:

- $(\ell, v) \stackrel{\delta}{\rightarrow}\left(\ell, v^{\prime}\right)$ if $v^{\prime}=v+R(\ell) \cdot \delta$ and $v^{\prime} \vDash I(\ell)$.

- $(\ell, v) \stackrel{a}{\rightarrow}\left(\ell^{\prime}, v^{\prime}\right)$ if $v \vDash g$ and there exists an edge $e \in E$ such that $e=$ $\left(\ell, g, a, Y, r, \ell^{\prime}\right), v^{\prime}=v[Y=0]+W(e) \cdot 1$ and $v^{\prime} \vDash I\left(\ell^{\prime}\right)$.

An (infinite) weighted word over actions $\mathcal{A}$ and clocks $C$ is a sequence $w=$ $\left(a_{0}, v_{0}\right)\left(a_{1}, v_{1}\right) \ldots$ of pairs of actions $a_{i} \in \mathcal{A}$ and valuations $v_{i} \in \mathcal{V}(C)$. For $i \geq 0$, we denote by $w^{i}$ the weighted word $w^{i}=\left(a_{i}, v_{i}\right)\left(a_{i+1}, v_{i+1}\right) \ldots$

A WTA $A=\left(L, \ell_{0}, C_{i}, C_{o}, E, W, I, R\right)$ over $\mathcal{A}$ generates a weighted word $w=\left(a_{0}, v_{0}\right)\left(a_{1}, v_{1}\right) \ldots$ over actions $\mathcal{A}$ and observable clocks $C_{o}$, iff $v_{0}=\overrightarrow{0}$ and there exists a sequence of transitions

$$
\left(\ell_{0}, v_{0}^{\prime}\right) \stackrel{\delta_{0}}{\longrightarrow}\left(\ell_{0}, v_{0}^{\prime \prime}\right) \stackrel{a_{0}}{\longrightarrow}\left(\ell_{1}, v_{1}^{\prime}\right) \stackrel{\delta_{1}}{\longrightarrow} \ldots \stackrel{a_{n}}{\longrightarrow}\left(\ell_{n+1}, v_{n+1}^{\prime}\right) \ldots,
$$

and for any $i$ the valuation $v_{i}$ is a projection of $v_{i}^{\prime}$ to $C_{o}$, i.e. $v_{i}(c)$ is equal to $v_{i}^{\prime}(c)$ for any observable clock $c \in C_{o}$.

Note, that since observable clocks are never reset and grow only with positive rates, the values of observable clocks can not decrease in a word generated by a WTA. In fact, we restrict ourselves to WTAs that generate cost-divergent words (i.e. for any observable clock $c$ and constant $k \in \mathbb{R}_{\geq 0}$ there is $v_{i}$ such, that $v_{i}(c)>$ $k$ ). If we consider that the WTA in Fig. 1(left) has only one observable clock c, then this WTA can generate a weighted word (ok, $\{\mathrm{c} \mapsto 2.0\}$ ), (problem, $\{\mathrm{c} \mapsto$ $3.1\}),($ cheap,$\{\mathrm{c} \mapsto 4.2\}), \ldots$.

We let $\mathcal{L}(A)$ denote the set of all weighted words generated by an WTA $A$ and refer to it as the language of $A$. 
A network of Weighted Timed Automata is a parallel composition of several WTA that have disjoint set of clocks and same set of actions $\mathcal{A}$. The automata are synchronized regarding discrete transitions such that if one automata performs a transition $\stackrel{a}{\rightarrow}$ all other also must perform an $\stackrel{a}{\rightarrow}$ transition. The notion of language recognized by WTA is naturally extended to the networks of Weighted Timed Automata.

In [10] we proposed a stochastic semantics for WTA, i.e. a probability measure over the set of accepted weighted words $\mathcal{L}(A)$. The non-determinism regarding discrete transitions for a single WTA is resolved using a uniform probabilistic choice among the possible transitions. Non-determinism regarding delays from a state $(\ell, v)$ of a single WTA is resolved using a density function $\mu_{(\ell, v)}$ over delays in $\mathbb{R}_{\geq 0}$ being either a uniform or an exponential distribution depending on whether the invariant of $\ell$ is empty or not.

The stochastic semantics for networks of WTA is then given in terms of repeated races between the component WTAs of the network: before a discrete transition each WTA chooses a delay according to its delay density function; then the WTA with a smallest delay wins the race and chooses probabilistically the action that the network must perform.

\subsection{Monitoring Weighted Timed Automata}

A monitoring weighted timed automaton (MWTA) $A_{M}$ is a special kind of WTA used to define allowed behavior of a given WTA $A$ (or a network of WTAs): a weighted word generated by $A$ is fed as input to $A_{M}$ for acceptance. For this, the actions of $A$ and $A_{M}$ coincide and there is a correspondence between the monitoring clocks of $A_{M}$ and the observable clocks $A$ ensuring that corresponding clocks grow with the same rate.

Definition 2. A Monitoring Weighted Timed Automaton (MWTA) over the clocks $C$ and the actions $\mathcal{A}$ is a tuple $\left(L, \ell_{0}, \ell_{a}, \ell_{r}, C_{M}, E, m\right)$ where:

$-L$ is a finite set of locations,

$-\ell_{0} \in L$ is the initial location,

$-\ell_{a} \in L$ and $\ell_{r} \in L$ are the accepting and rejecting locations, correspondingly,

$-C_{M}$ is a finite set of local clocks,

- $E \subseteq\left(L \backslash\left\{l_{a}, l_{r}\right\}\right) \times \mathcal{A} \times 2^{\mathcal{B}\left(C_{M}\right)} \times 2^{C_{M}} \times L$ is a finite set of edges,

- $m: C_{M} \rightarrow C$ gives the correspondence of local clocks and $C$.

We assume, that MWTAs are complete, i.e. for any location $l \in L \backslash\left\{l_{a}, l_{r}\right\}$, action $a \in \mathcal{A}$ and valuation $v \in \mathcal{V}\left(C_{M}\right)$ there exists an edge $\left(l, a, g, Y, l^{\prime}\right) \in E$ such that $v \models g$. An MWTA is called deterministic if not more than one such edge exists.

An MWTA $A_{M}=\left(L, \ell_{0}, \ell_{a}, \ell_{r}, C_{M}, E, m\right)$ over clocks $C$ and actions $\mathcal{A}$ accepts a weighted word $\left(a_{0}, v_{0}\right)\left(a_{1}, v_{1}\right) \ldots$ over the same $C$ and $\mathcal{A}$, iff there exists a finite sequence $\left(l_{0}, v_{0}^{\prime}\right),\left(l_{1}, v_{1}^{\prime}\right), \ldots,\left(l_{n}, v_{n}^{\prime}\right)$ of states of $A_{M}$ such, that:

$-v_{0}^{\prime}(c)=v_{0}(m(c))$ for any clock $c \in C_{M}$, 
- for any $i$ there exists an edge $\left(l_{i}, a_{i}, g_{i}, Y_{i}, l_{i+1}\right) \in E$ such, that:

- $v_{i}^{\prime} \models g_{i}$ and

- for every clock $c \in C_{M}$, if $c \in Y_{i}$ then $v_{i+1}^{\prime}(c)=0$, and otherwise $v_{i+1}^{\prime}(c)=v_{i}^{\prime}(c)+\left(v_{i+1}(m(c))-v_{i}(m(c))\right)$,

$-l_{n}=l_{a}$ is the accepting location of A.

Thus, after reading an element of an input weighted word, a local clock $c$ the MWTA either reset, or it grows with the same rate as the corresponding clock $m(c)$ in the input word.

\subsection{Weighted Metric Temporal logic $\mathrm{WMTL}_{\leq}$}

Definition 3. [7] A $W M T L_{\leq}$formula $\varphi$ over atomic propositions $P$ and clocks $C$ is defined by the grammar

$$
\varphi::=p|\neg \varphi| \varphi_{1} \wedge \varphi_{2}|O \varphi| \varphi_{1} \mathrm{U}_{\leq d}^{c} \varphi_{2}
$$

where $p \in P, d \in \mathbb{N}$, and $c \in C$.

Let false be an abbreviation for $(p \wedge \neg p)$, and true be an abbreviation for $\neg$ false. The other commonly used operators in $\mathrm{WMTL}_{\leq}$can be defined by the following abbreviations: $\left(\varphi_{1} \vee \varphi_{2}\right)=\neg\left(\neg \varphi_{1} \wedge \neg \varphi_{2}\right),\left(\varphi_{1} \rightarrow \varphi_{2}\right)=\left(\neg \varphi_{1}\right) \vee \varphi_{2}$, $\diamond_{\leq d}^{c} \varphi=$ true $\mathrm{U}_{\leq d}^{c} \varphi, \square_{\leq d}^{c} \varphi=\neg \diamond_{\leq d}^{c} \neg \varphi$, and $\varphi_{1} \mathrm{R}_{\leq d}^{c} \varphi_{2}=\neg\left(\neg \varphi_{1} \mathrm{U}_{\leq d}^{c} \neg \varphi_{2}\right)$, where $\mathrm{R}$ is the "release" operator. We also assume, that there always exists a special clock $\tau \in C$ (that grows with a rate 1 in an automaton being monitored).

Assuming that $P$ are atomic propositions over actions $\mathcal{A}, \mathrm{WMTL}_{\leq}$formulas are interpreted over weighted words. For a given weighted word $w=$ $\left(a_{0}, v_{0}\right)\left(a_{1}, v_{1}\right)\left(a_{2}, v_{2}\right) \ldots$ over $\mathcal{A}$ and $C$ and $\mathrm{WMTL}_{\leq}$formula $\varphi$ over $P$ and $C$, the satisfaction relation $w^{i} \models \varphi$ is defined inductively:

1. $w^{i} \models p$ iff $a_{i}=p$

2. $w^{i} \models \neg \varphi$ iff $w^{i} \not \models \varphi$

3. $w^{i}=O \varphi$ iff $w^{i+1}=\varphi$

4. $w^{i} \models \varphi_{1} \wedge \varphi_{2}$ iff $w^{i} \models \varphi_{1}$ and $w^{i} \models \varphi_{2}$

5. $w^{i} \models \varphi_{1} \mathrm{U}_{\leq d}^{c} \varphi_{2}$ iff there exists $j$ such that $j \geq i, w^{j} \models \varphi_{2}, v_{j}(c)-v_{i}(c) \leq d$, and $w^{k} \models \varphi_{1}$ for all $k$ with $i \leq k<j$.

We say, that a weighted word $w$ satisfies $\varphi$, iff $w^{0}=\varphi$, and denote by $\mathcal{L}(\varphi)$ the set of all weighted words that are satisfied by $\varphi \cdot \varphi_{1}$ and $\varphi_{2}$ are equivalent if they are satisfied by the same weighted words, in which case we write $\varphi_{1} \equiv \varphi_{2}$.

Given the stochastic semantics of a WTA $A$, and semantics of $\mathrm{WMTL}_{\leq}$ formula $\varphi$, we can define $\operatorname{Pr}[A \models \varphi]$ to be the probability that a random run of $A$ satisfies $\varphi$. This probability is well-defined because $\mathcal{L}(A) \cap \mathcal{L}(\varphi)$ is a countable union and intersection of measurable sets and thus it is measurable itself. 


\section{From Formulas to Monitors}

In this section we present a novel procedure for translating $\mathrm{WMTL}_{\leq}$formulas into equivalent MWTA monitors, providing an essential and efficient component of our tool-chain. However, to enable monitor-based, statistical model checking it is essentially that the generated MWTA is deterministic. Unfortunately, this might not always be possible as there are $\mathrm{WMTL}_{\leq}$formulas for which no equivalent deterministic MWTA exist ${ }^{4}$. As a remedy, we describe how basic syntactic transformations prior to translation allow us to obtain deterministic over- and under-approximating MWTAs for any given formula $\varphi$. In Section 5, we shall see that these approximations are tight and often exact.

\subsection{Closures \& Extended Formulas}

In this section, we assume that $\varphi$ is $\mathrm{WMTL}_{\leq}$formula over propositions $P$ and (observable) clocks $C$ and has been transformed into negative normal form $(\mathrm{NNF})$, i.e. an equivalent formula in which negations are applied to the atomic propositions only. We use $\operatorname{Sub}(\varphi)$ to denote all the sub-formulas of $\varphi$.

In order to further expand $\varphi$ into a disjunctive normal form, we introduce for each $\phi_{1} \mathrm{U}_{\leq d}^{c} \phi_{2} \in \operatorname{Sub}(\varphi)$ and each $\phi_{1} \mathrm{R}_{\leq d}^{c} \phi_{2} \in \operatorname{Sub}(\varphi)$, one local clock $x$ and two timing constraints $x \leq d$ and $x>d$ to express some timing information related to $\phi_{1} \mathrm{U}_{\leq d}^{c} \phi_{2}$ and $\phi_{1} \mathrm{R}_{\leq d}^{c} \phi_{2}$. Also, we introduce auxiliary formulas $\phi_{1} \mathrm{U}_{\leq d-x}^{c} \phi_{2}$ and $\phi_{1} \mathrm{R}_{\leq d-x}^{\bar{c}} \phi_{2}$ to express some requirements that should be satisfied in the future when we try to guarantee $\phi_{1} \mathrm{U}_{\leq d}^{c} \phi_{2} \in \operatorname{Sub}(\varphi)$ or $\phi_{1} \mathrm{R}_{\leq d}^{c} \phi_{2} \in \operatorname{Sub}(\varphi)$ is true in the current state.

We define $X_{\varphi}=\left\{x_{\phi_{1} \cup_{\leq d}^{c} \phi_{2}} \mid \phi_{1} \bigcup_{\leq d}^{c} \phi_{2} \in \operatorname{Sub}(\varphi)\right\} \cup\left\{x_{\phi_{1} \mathrm{R}_{\leq d}^{c} \phi_{2}} \mid \phi_{1} \mathrm{R}_{\leq d}^{c} \phi_{2} \in\right.$ $\operatorname{Sub}(\varphi)\}$ to be the set of all local clocks for $\varphi$, where $x_{\phi_{1} \mathrm{U}_{\leq d}^{c} \phi_{2}}$ is the clock assigned to $\phi_{1} \mathrm{U}_{\leq d}^{c} \phi_{2}$ and $x_{\phi_{1} \mathrm{R}_{\leq d}^{c} \phi_{2}}$ is the local clock assigned to $\phi_{1} \mathrm{R}_{\leq d}^{c} \phi_{2}$. We call $x_{\phi_{1} \mathrm{U}_{\leq d}^{c} \phi_{2}}$

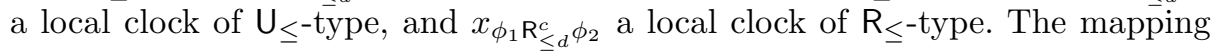
$m$ from local clocks $X_{\varphi}$ to observable clocks $C$ is defined by $m\left(x_{\phi_{1} \mathrm{U}_{\leq d}^{c} \phi_{2}}\right)=c$ and $m\left(x_{\phi_{1} \mathrm{R}_{<d}^{c} \phi_{2}}\right)=c$. The closure of $\varphi$, write as $\operatorname{CL}(\varphi)$, is now defined by the following rules:

1. true $\in \operatorname{CL}(\varphi), \operatorname{Sub}(\varphi) \subseteq \operatorname{CL}(\varphi)$

2. If $\phi_{1} \mathrm{U}_{\leq d}^{c} \phi_{2} \in \operatorname{Sub}(\varphi)$ and $x$ is the local clock assigned to $\phi_{1} \mathrm{U}_{\leq d}^{c} \phi_{2}$, then $x \leq d, \bar{x}>d, \phi_{1} \mathrm{U}_{<d-x}^{c} \phi_{2} \in \mathrm{CL}(\varphi)$

3. If $\phi_{1} \mathrm{R}_{\leq d}^{c} \phi_{2} \in \operatorname{Sub}(\varphi)$ and $x$ is the local clock assigned to $\phi_{1} \mathrm{R}_{\leq d}^{c} \phi_{2}$, then $x \leq d, x>d, \phi_{1} \mathrm{R}_{<d-x}^{c} \phi_{2} \in \mathrm{CL}(\varphi)$

4. If $\Phi_{1}, \Phi_{2} \in \mathrm{CL}(\varphi)$, then $\Phi_{1} \wedge \Phi_{2}, \Phi_{1} \vee \Phi_{2} \in \mathrm{CL}(\varphi)$

Obviously, $\mathrm{CL}(\varphi)$ has only finitely many different non-equivalent formulas.

For a local clock $x$, we use $\operatorname{rst}(x)$ to represent that $x$ will be reset at current step and unch $(x)$ to represent that $x$ will not be reset at current step. The set of extended formulas for $\varphi$, write as $\operatorname{Ext}(\varphi)$, is now defined by the following rules:

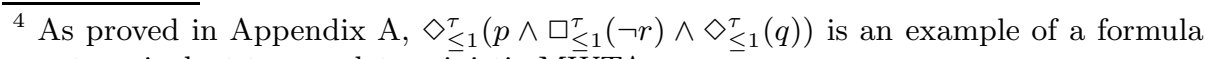
not equivalent to any deterministic MWTA. 
1. If $\Phi \in \mathrm{CL}(\varphi)$, then $\Phi, \mathrm{O} \Phi \in \operatorname{Ext}(\varphi)$

2. If $x \in X_{\varphi}$ is a local clock of $\mathrm{U}_{\leq}$-type, then $\operatorname{unch}(x) \in \operatorname{Ext}(\varphi)$

3. If $x \in X_{\varphi}$ is a local clock of $\mathrm{R}_{\leq}$-type, then $\operatorname{rst}(x) \in \operatorname{Ext}(\varphi)$

4. If $\Phi_{1}, \Phi_{2} \in \operatorname{Ext}(\varphi)$, then $\Phi_{1} \wedge \Phi_{2}, \Phi_{1} \vee \Phi_{2} \in \operatorname{Ext}(\varphi)$

Extended formulas can be interpreted using extended weighted words. An extended weighted word $\omega=\left(a_{0}, v_{0}, \nu_{0}\right)\left(a_{1}, v_{1}, \nu_{1}\right)\left(a_{2}, v_{2}, \nu_{2}\right) \ldots$ is a sequence where $w=\left(a_{0}, v_{0}\right)\left(a_{1}, v_{1}\right)\left(a_{2}, v_{2}\right) \ldots$ is a weighted word over $2^{P}$ and $C$, and for every $i \in \mathbb{N}, \nu_{i}$ is a clock valuation over $X_{\varphi}$ such that for all $x \in X_{\varphi}$, either $\nu_{i+1}(x)=v_{i+1}(m(x))-v_{i}(m(x))$ or $\nu_{i+1}(x)=\nu_{i}(x)+v_{i+1}(m(x))-v_{i}(m(x))$.

The semantics for extended formulas is naturally induced by the semantics of $\mathrm{WMTL}_{\leq}$formulas:

Definition 4. Let $\omega=\left(a_{0}, v_{0}, \nu_{0}\right)\left(a_{1}, v_{1}, \nu_{1}\right)\left(a_{2}, v_{2}, \nu_{2}\right) \ldots$ be an extended weighted word and $\Phi \in \operatorname{Ext}(\varphi)$. The satisfaction relation $\omega^{i} \models_{e} \Phi$ is inductively defined as follows:

1. $\omega^{i}=_{e} x \sim d$ iff $\nu_{i}(x) \sim d$

2. $\omega^{i}==_{e} r s t(x)$ iff $\nu_{i+1}(x)=v_{i+1}(m(x))-v_{i}(m(x))$

3. $\omega^{i}={ }_{e} \operatorname{unch}(x)$ iff $\nu_{i+1}(x)=\nu_{i}(x)+v_{i+1}(m(x))-v_{i}(m(x))$

4. $\omega^{i}==_{e} \phi$ iff $w^{i} \models \phi$, if $\phi \in \operatorname{Sub}(\varphi)$

5. $\omega^{i} \models{ }_{e} \varphi_{1} \cup_{\leq d-x}^{c} \varphi_{2}$ iff there exists $j$ such that $j \geq i, w^{j} \models \varphi_{2}, v_{j}(c)-v_{i}(c) \leq$ $d-\nu_{i}(x)$, and $w^{k} \models \varphi_{1}$ for all $k$ with $i \leq k<j$

6. $\omega^{i} \models_{e} \varphi_{1} \mathrm{R}_{\leq d-x}^{c} \varphi_{2}$ iff for all $j \geq i$ such that $v_{j}(c)-v_{i}(c) \leq d-\nu_{i}(x)$, either $w^{j} \models \varphi_{2}$ or there exists $k$ with $i \leq k<j$ and $w^{k} \models \varphi_{1}$

7. $\omega^{i} \models_{e} \Phi_{1} \wedge \Phi_{2}$ iff $\omega^{i} \models_{e} \Phi_{1}$ and $\omega^{i} \models_{e} \Phi_{2}$

8. $\omega^{i}==_{e} \Phi_{1} \vee \Phi_{2}$ iff $\omega^{i} \models_{e} \Phi_{1}$ or $\omega^{i} \models{ }_{e} \Phi_{2}$

9. $\omega^{i} \models_{e} O \Phi$ iff $\omega^{i+1} \models_{e} \Phi$

$\omega^{i}$ is a model of $\Phi$ if $\omega^{i} \models_{e} \Phi$ and two extended $W M T L_{\leq- \text {formulas are said }}$ equivalent if they have exactly the same models.

\subsection{Constructing Non-deterministic Monitors}

As in the construction of Büchi automata from LTL formulas, we will break a formula into a disjunction of several conjunctions [9]. Each of the disjuncts corresponds to a transition of a resulting observer automaton and specifies the requirements to be satisfied in the current and in the next states. In the rest of this section, we use $r s t\left(\left\{x_{1}, x_{2}, \ldots, x_{n}\right\}\right)$ and $\operatorname{unch}\left(\left\{y_{1}, y_{2}, \ldots, y_{n}\right\}\right)$ to denote the formula of $r s t\left(x_{1}\right) \wedge r s t\left(x_{2}\right) \wedge \ldots \wedge r s t\left(x_{n}\right)$ and the formula of unch $\left(y_{1}\right) \wedge$ unch $\left(y_{2}\right) \wedge \ldots \wedge$ unch $\left(y_{n}\right)$ respectively. A basic conjunction is an extended formula of the form:

$$
\alpha \wedge g \wedge r s t(X) \wedge \operatorname{unch}(Y) \wedge O(\Psi)
$$

where $\alpha$ is a conjunction of literals (a literal is a proposition or its negation), $g$ is a conjunction of clock constraints, $X$ is a set of local clocks with $\mathrm{R}_{\leq}$-type, $Y$ is a set of local clocks with $\mathrm{U}_{\leq}$-type, and $\Psi$ is a formula in $\mathrm{CL}(\varphi) . \alpha \wedge g \wedge r \operatorname{st}(X) \wedge u n \operatorname{ch}(Y)$ 
specifies the requirements to be satisfied in the current state and $\Psi$ specifies the requirements in the next-state. The next Lemma 1 and main Theorem 1 provides the construction of a monitor from a formula. A full proof is given in Appendix D.

Lemma 1. Each formula in $C L(\varphi)$ can be transformed into a disjunction of several basic conjunctions by using the following rules and Boolean equivalences.

1. $f \mathrm{U}_{\leq d}^{c} g=g \vee\left(f \wedge O\left((x \leq d) \wedge\left(f \mathrm{U}_{\leq d-x}^{c} g\right)\right)\right)$, where $x$ is the clock assigned to $f \mathrm{U}_{\leq d}^{\bar{c}} g$

2. $f \mathrm{U}_{\leq d-x}^{\bar{c}} g=g \vee\left(f \wedge \operatorname{unch}(x) \wedge O\left((x \leq d) \wedge\left(f \mathrm{U}_{\leq d-x}^{c} g\right)\right)\right)$

3. If $f \overline{\mathrm{R}}_{\leq d}^{c} g=g \wedge\left(f \vee\left(r s t(x) \wedge O\left(\left((x \leq d) \wedge\left(f \mathrm{R}_{\leq d-x}^{c} g\right)\right) \vee(x>d)\right)\right)\right)$, where $x$ is the clock assigned to $f \mathrm{R}_{\leq d}^{c} g$

4. $f \mathrm{R}_{\leq d-x} g=g \wedge\left(f \vee O\left(\left((x \leq d) \wedge\left(f \mathrm{R}_{\leq d-x} g\right)\right) \vee(x>d)\right)\right)$

5. $(O \bar{f}) \wedge(O g)=O(f \wedge g)$

6. $(O f) \vee(O g)=O(f \vee g)$

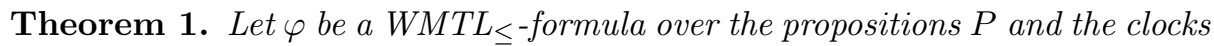
$C$ and is in NNF. Let the $M W \bar{T} A A_{\varphi}=\left(L, \ell_{0}, \ell_{a}, C_{M}, E, m\right)$ over the clocks $C$ and the actions $\mathcal{A}=2^{P}$ be defined as follows:

$-L=\{\{\phi\} \mid \phi \in C L(\varphi)\}$ is a finite set of locations, and $\ell_{0}=\{\varphi\}$ is the initial location;

$-\ell_{a}=\{$ true $\}$ is the accepting location;

$-C_{M}=X_{\varphi}$ is the set of all local clocks for $\varphi$;

$-\left(\left\{f_{1}\right\}, a, g, \lambda,\left\{f_{2}\right\}\right) \in E$ iff $\alpha \wedge g \wedge \operatorname{rst}(X) \wedge$ unch $(Y) \wedge O\left(f_{2}\right)$ is a basic conjunction of $f_{1}$ and that a satisfies $\alpha$, and for each $x \in X_{\varphi}$ of $\mathrm{U}_{\leq}$-type, $x \in \lambda$ iff $x \notin Y$, and for each $x \in X_{\varphi}$ of $\mathrm{R}_{\leq- \text {type, }} x \in \lambda$ iff $x \in X$;

$-m$ is defined by $m\left(x_{\phi_{1} \mathrm{U}_{\leq d}^{c} \phi_{2}}\right)=c$ and $m\left(x_{\phi_{1} \mathrm{R}_{\leq d}^{c} \phi_{2}}\right)=c$.

Then $L(\varphi)=L\left(A_{\varphi}\right)$.

Example 1. Fig.2a is a MWTA obtained with our approach for $f=\left(\diamond_{\leq 1}^{x} p\right) \vee$ $\left(\square_{\leq 2}^{c} q\right)=\left(\right.$ true $\left.\mathrm{U}_{\leq 1}^{x} p\right) \vee\left(\right.$ false $\left.\mathrm{R}_{\leq 2}^{c} q\right)$.

\subsection{Constructing Deterministic Monitors}

The construction of the section 3.2 might produce non-deterministic automata. In fact, as stated earlier, there exist $\mathrm{WMTL}_{\leq}$formulas for which no equivalent deterministic MWTA. To get deterministic MWTA for $\mathrm{WMTL}_{\leq- \text {formulas, we }}$ further translate formulas in disjunctive into the following deterministic form by repeated use of the logical equivalence $p \Leftrightarrow(p \wedge q) \vee(p \wedge \neg q)$.

$$
F=\bigvee_{i=1}^{n}\left(\alpha_{i} \wedge g_{i} \wedge \bigvee_{k=1}^{i_{k}}\left(\operatorname{rst}\left(X_{i k}\right) \wedge \operatorname{unch}\left(Y_{i k}\right) \wedge O\left(\Psi_{i k}\right)\right)\right)
$$




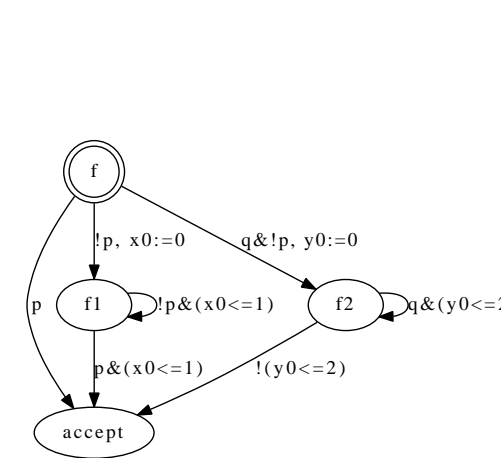

(a) Non-deterministic monitor

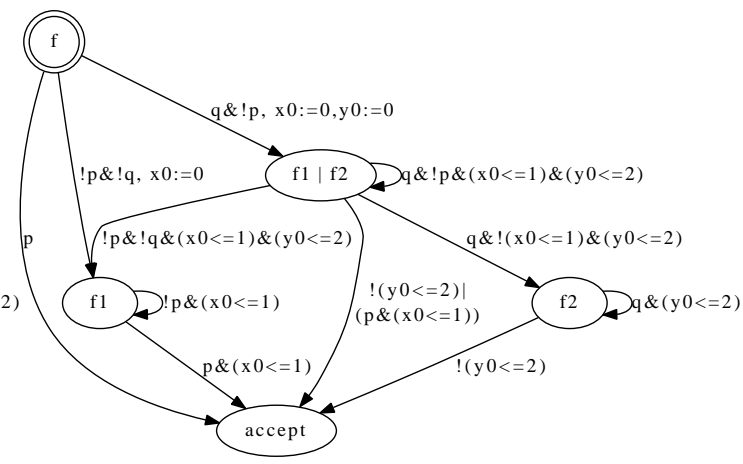

(b) Deterministic under-approximation monitor

Fig. 2: Monitoring WTA for $f \equiv\left(\diamond_{\leq 1}^{x} p\right) \vee\left(\square^{y}{ }_{\leq 2} q\right)$, with $f 1 \equiv(x 0 \leq 1) \wedge\left(\right.$ true $\left.\mathrm{U}_{\leq 1-x 0}^{x} p\right)$ and $f 2 \equiv\left((c 0 \leq 2) \wedge\left(\right.\right.$ false $\left.\left.\mathrm{R}_{\leq 2-y 0}^{y} q\right)\right) \vee(y 0>2)$.

where $X_{i k} \subseteq X_{\varphi}$ is a set of local clocks of type $\mathrm{R}_{\leq}$and $Y_{i k} \subseteq X_{\varphi}$ is a set of local clocks of type $\mathrm{U}_{\leq}$, and for all $i \neq j: \alpha_{i} \wedge g_{i} \wedge \alpha_{j} \wedge g_{j}$ is false.

Using the facts that $O$ distributes over $\vee$, and $\operatorname{rst}(X)$ and $u n c h(X)$ are monotonic in $X$, the following formulas are obviously strengthened $\left(F^{u}\right)$ respectively weakened $\left(F^{o}\right)$ versions of $F$ :

$$
\begin{aligned}
F^{u} & =\bigvee_{i=1}^{n}\left(\alpha_{i} \wedge g_{i} \wedge \operatorname{rst}\left(\bigcup_{k=1}^{i_{k}} X_{i k}\right) \wedge \operatorname{unch}\left(\bigcup_{k=1}^{i_{k}} Y_{i k}\right) \wedge O\left(\bigvee_{k=1}^{i_{k}} \Psi_{i k}\right)\right) \\
F^{o} & =\bigvee_{i=1}^{n}\left(\alpha_{i} \wedge g_{i} \wedge \operatorname{rst}\left(\bigcap_{k=1}^{i_{k}} X_{i k}\right) \wedge \operatorname{unch}\left(\bigcap_{k=1}^{i_{k}} Y_{i k}\right) \wedge O\left(\bigvee_{k=1}^{i_{k}} \Psi_{i k}\right)\right)
\end{aligned}
$$

Interestingly, by simply applying the construction of Theorem 1 to $F^{u}\left(F^{o}\right)$ we immediately obtain a deterministic under-approximating (over-approximating) $\operatorname{MWTA} A_{\varphi}^{u}\left(A_{\varphi}^{o}\right)$ for $\varphi$.

Example 2. (continued) Fig.2b is the under-approximation deterministic MWTA for $f=\left(\diamond_{\leq 1}^{x} p\right) \vee\left(\square_{\leq 2}^{c} q\right)$.

\section{The Tool Chain}

Figure 3 provides an architectural view of our tool chain. The tool chain takes as input an $\mathrm{MITL}_{\leq}$formula $\varphi$, a WTA model $M$, as well as statistical parameters $\epsilon, \alpha$ for controlling precision and confidence level. As a result a confidence interval for the probability $\operatorname{Pr}[M \models \varphi]$ with the desired precision and confidence level is returned. 


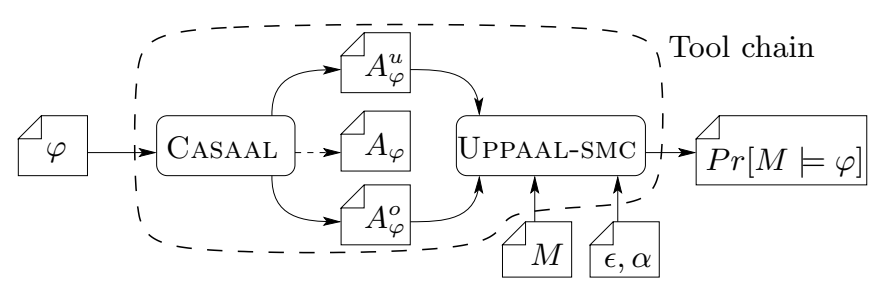

Fig. 3: Tool chain architecture

Casaal The tool chain includes the new tool component CASAAL for generating monitors. The tool is implemented in $\mathrm{C}++$ and is build on top of the $\mathrm{Spot}^{5}$ opensource library for LTL to Büchi automata translation. We also use Buddy ${ }^{6} \mathrm{BDD}$ package to handle operations over Boolean formulas. Given a MITL $\leq$ formula $\varphi$, CASAAL may construct an exact monitoring WTA $A_{\varphi}$, as well as two - possibly approximating - monitoring WTAs, $A_{\varphi}^{u}$ and $A_{\varphi}^{o}$. Table 1 demonstrates some experimental results for CASAAL. The formulas were also used in [13] and for comparison we list their results as well. More experimental results are given in Appendix C.

\begin{tabular}{|c|r|r|r|r|}
\hline formula & automaton & states & trans & time(s) \\
\hline & nondet & 5 & 14 & 0.02 \\
\cline { 2 - 5 } & under & 9 & 58 & 0.02 \\
\cline { 2 - 5 } & over & 9 & 56 & 0.04 \\
\cline { 2 - 5 } & Geilen & 14 & 30 & \\
\hline \hline \multirow{3}{*}{$\left(p \rightarrow \mathrm{U}_{\leq 1}^{\tau}\left(q \mathrm{U}_{\leq 1}^{\tau}\left(\mathrm{R}_{\leq 1}^{\tau} s\right)\right)\right.$} & nondet & 7 & 19 & 0.01 \\
\cline { 2 - 5 } & under & 9 & 32 & 0.01 \\
\cline { 2 - 5 } & over & 9 & 32 & 0.01 \\
\cline { 2 - 5 } & Geilen & 21 & 64 & \\
\hline \hline & nondet & 17 & 121 & 0.02 \\
\cline { 2 - 5 } & under & 17 & 121 & 0.03 \\
\cline { 2 - 5 } & over & 17 & 121 & 0.03 \\
\cline { 2 - 5 } & Geilen & 60 & 271 & \\
\hline
\end{tabular}

Table 1: Experimental results for $\mathrm{WMTL}_{\leq}$formulas.

Uppaal-smc $[10,11]$ is a tool that allows to estimate and test $\operatorname{Pr}[M \models \phi]$, i.e. the probability that a random run of a given WTA model $M$ satisfies $\phi$, where $\phi$ is a $\mathrm{WMTL}_{\leq}$formula restricted to the form $\diamond_{\leq d}^{c} \psi$ and $\psi$ is a state predicate. Estimation is performed by generating a number of random simulations of $M$, where each simulation stops when either it reaches a state when $\psi$ is satisfied, or $c \leq d$ is violated.

Combining Casaal and Uppaal-smc Let us describe how we use UPPAALSMC together with the CASAAL tool to estimate the probability that a random run of a WTA model $M$ satisfies a general $\mathrm{WMTL}_{\leq}$property $\phi$, i.e. $\operatorname{Pr}[M \models \phi]$.

\footnotetext{
${ }^{5}$ http://spot.lip6.fr/wiki/

${ }^{6}$ http://sourceforge.net/projects/buddy/develop
} 
Let us first assume, that one of two deterministic approximations for $\varphi$ returned by CASAAL is exact. This means, that we have MWTA $A_{\varphi}^{\text {det }}=\left(L, \ell_{0}, \ell_{a}\right.$, $\left.\ell_{r}, C_{M}, E, m\right)$ such that $\mathcal{L}\left(A_{\varphi}^{\text {det }}\right)=\mathcal{L}(\varphi)$. First, we augment MWTA $A_{\varphi}^{\text {det }}$ with a clock $c^{\dagger}$ that will grow with rate 1 in rejecting location $\ell_{r}$, and with rate 0 in all other locations. Additionally, for every clock $c \in C_{M}$ we duplicate all rates and transition weights from the corresponding clock $m(c)$ to make sure, that the clocks of $A_{\varphi}^{\text {det }}$ grow with the same rate as the corresponding clocks of the automaton $M$ being monitored. Forming a parallel composition of $M$ and $A_{\varphi}^{\text {det }}$, we may now use UPPAAL-SMC to estimate the probability $p=\operatorname{Pr}\left[M \| A_{\varphi}^{\text {det }} \models \diamond_{\leq 1}^{c^{\dagger}}\left(\ell_{a}\right)\right]$. This can be done because of the following theorem:

Theorem 2. If $M$ produces cost-divergent runs only, then each simulation of $M \| A_{\varphi}^{\text {det }}$ will end up in accepting or rejecting location of $A_{\varphi}^{\text {det }}$ after finite number of steps.

If none of the two MWTAs $A_{\varphi}^{o}$ and $A_{\varphi}^{u}$ are exact determinization of $A_{\varphi}$ (i.e. $\mathcal{L}\left(A_{\varphi}^{u}\right) \subsetneq \mathcal{L}(\varphi) \subsetneq \mathcal{L}\left(A_{\varphi}^{o}\right)$ ), then we use both of them to compute upper (using $A_{\varphi}^{o}$ ) and lower (using $A_{\varphi}^{u}$ ) bounds for $\operatorname{Pr}[M \models \varphi]$. Indeed, if $n_{1}\left(n_{2}\right.$, correspondingly) out of $m$ random simulations of $M \| A_{\varphi}^{u}\left(M \| A_{\varphi}^{o}\right.$, correspondingly) ended in accepting location $l_{a}^{u}\left(l_{a}^{o}\right.$, correspondingly), then with significance level of $\alpha$ we can accept a hypothesis $H_{1}\left(H_{2}\right.$, correspondingly) that $\operatorname{Pr}[M \models \varphi] \geq n_{1} / m-\varepsilon$ $\left(\operatorname{Pr}[M \models \varphi] \leq n_{2} / m+\varepsilon\right)$. By combining hypothesis $H_{1}$ and $H_{2}$ we can obtain a confidence interval $\left[n_{1} / m-\varepsilon, n_{2} / m+\varepsilon\right]$ for $\operatorname{Pr}[M \models \varphi]$ with significance level of $1-(1-\alpha)^{2}=2 \alpha-\alpha^{2}$.

\section{Case Studies}

We performed several case studies to demonstrate the applicability of our tool chain. In the first case study we analyze the performance of CASAAL on a set of randomly generated $\mathrm{WMTL}_{\leq}$formulas. In the second case study we use a model of a robot moving on a two-dimensional grid, this model was first analyzed in [5] using the manually constructed monitoring timed automaton.

Finally, in the appendix B we demonstrate the scalability of our tool chain by applying it to the analysis of a real-world IEEE 802.15.4 CSMA/CA protocol.

\subsection{Automatically Generated Formulas}

In the first case study we analyze the performance of CASAAL on a set of randomly generated $\mathrm{WMTL}_{\leq}$formulas. We generated 1000 formulas with 2, 3 and 4 actions, and created deterministic over and approximations for these formulas. Each of the formulas have 15 connectives (release, until, conjunction or disjunction) and four clocks.

For the formulas where only one or none of the approximations was exact, we measured the "stochastic difference" between approximations by generating a number of random weighted words and estimating the probability that the over approximation accepts a random word, when the under approximation does not. 
Table 2 reports the amount of formulas for which the under or over approximation was exact and the amount of formulas where none of them was exact. It also contains the average time spent for generating the monitors and the average number of locations, and the stochastic difference.

\subsection{Robot Control}

We consider the case of a robot moving on a twodimensional grid that was explored in e.g. [5]. Each field of the grid is either normal, on fire, cold as ice or it is a wall which that cannot be passed. Also, there is a goal field that the robot must reach. The robot is moving in a random fashion i.e. it stays in a field for some time, and then randomly moves to one of the neighboring fields (if it is not a wall). Fig. 5 shows a robot controller implementing this along with the grid we use.

We are interested in the probability that the

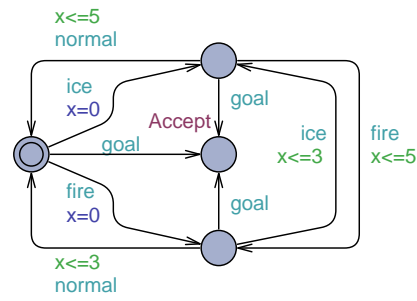

Fig. 4: Observer automaton used in [5] robot reaches its goal location without staying on consecutive fire fields for more than one time units and on consecutive ice fields for more than two time units.

In [5] the authors solved this problem by manually constructing a monitoring automaton to operate in parallel with the model of the robot. The automaton they used is depicted in Figure 4. Using $\mathrm{WMTL}_{\leq}$we can express the same requirement more easily as $\varphi \equiv\left(\varphi_{1} \wedge \varphi_{2}\right) \mathrm{U}_{\leq 10}^{\tau}$ goal, where:

$$
\begin{aligned}
& \varphi_{1} \equiv \text { ice } \Longrightarrow \diamond_{\leq 2}^{\tau}(\text { fire } \vee \text { normal } \vee \text { goal }) \\
& \varphi_{2} \equiv \text { fire } \Longrightarrow \diamond_{\leq 1}^{\tau}(\text { ice } \vee \text { normal } \vee \text { goal })
\end{aligned}
$$

CASAAL produces an MWTA (6 locations, 55 edges) that is an exact underapproximation for $\varphi$. Based on this MWTA, our tool chain estimates the probability that the random behavior of the robot satisfies $\varphi$ to lie in the interval $[0.373,0.383]$ with a confidence of $95 \%$. Fig. $5 \mathrm{c}$ shows how we can visualize and compare the different distributions using the plot composer of UPPAAL-SMC.

Energy We extend the model by limiting the energy of the robot that will stop moving when it runs out of energy. Furthermore, it can regain energy while staying on fire fields and use additional energy while staying on ice fields. Let $c$ be the clock accumulating the amount of consumed energy. Now, we can express the property $\varphi \equiv\left(\varphi_{1} \wedge \varphi_{2} \wedge \neg\right.$ noEnergy $) \bigcup_{\leq 10}^{c}$ goal that the robot should not use

\begin{tabular}{|r|r|r|r|r|r|r|r|r|r|r|}
\cline { 2 - 11 } \multicolumn{1}{c|}{} & \multicolumn{4}{c|}{ \# exact } & \multicolumn{2}{|c|}{ Avg. time (s) } & \multicolumn{2}{c|}{ Avg. size } & \multicolumn{2}{|c|}{ Stochastic difference } \\
\hline Actions & under & over & none & one & under & over & under & over & no exact & one exact \\
\hline 2 & 831 & 542 & 169 & 289 & 0.24 & 1.01 & 6.35 & 6.35 & 0.27 & 0.15 \\
\hline 3 & 706 & 370 & 294 & 336 & 1.42 & 2.75 & 12.29 & 12.29 & 0.05 & 0.03 \\
\hline 4 & 586 & 233 & 414 & 353 & 8.66 & 13.05 & 22.97 & 22.97 & 0.01 & 0.02 \\
\hline
\end{tabular}

Table 2: Results for the random generated formula test. 


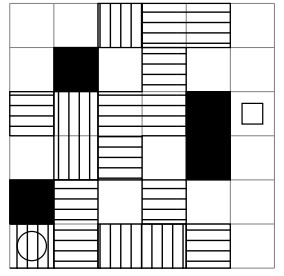

(a)

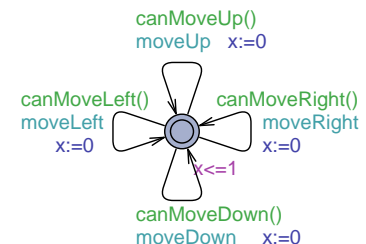

(b)

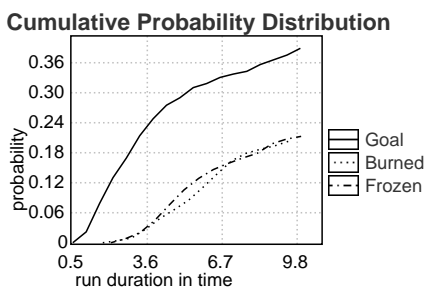

(c)

Fig. 5: (a) A $6 \times 6$ grid. The black fields are walls, the fields with vertical lines are on fire and the fields with horizontal lines contain ice. The circle indicates the robot's starting position and the square the goal.

(b) WTA implementing the random movement of the robot.

(c) Cumulative distribution of the robot reaching the goal, staying too long in the fire or too long on the ice.

more than 5 units of energy while obeying the requirements from before. The tool chain estimates the probability that the robot satisfies this requirement to lie in $[0.142 ; 0.152]$ with a confidence of $95 \%$.

\section{$6 \quad$ Related and Future Work}

To our knowledge, we are the first to propose and implement an algorithm for translation of $\mathrm{WMTL}_{\leq}$formulas into monitoring automata. However, if we level down to $\mathrm{MITL}_{\leq}$, there are several translation procedures described in the literature that are dealing with this logic. First, Rajeev Alur in [3] presents a procedure that is mostly theoretical and is not intended to be practically implemented. Second, Oded Maler et al. [16] proposed a procedure to translate MITL into temporal testers (not the classic timed automata), their procedure also has not been implemented. Nir Piterman et al. [17] proposed an approach how to translate MTL to deterministic timed automata under finite variability assumption (this assumption is not valid for the WTA stochastic semantics that we use). Finally, Marc Geilen[12] has implemented a procedure to translate MITL $_{\leq}$ to timed automata, but his approach works in continuous time semantics.

For future work we aim at extending our monitor- and approximate determinization constructions to $\mathrm{WMTL}_{[a, b]}$ with (non-singleton) cost interval-bounds on the $\mathrm{U}$ modality in order to allow for SMC for this more expressive logic. Here a challenge will be how to bound the length of the random runs to be generated.

\section{References}

1. G. Agha, J. Meseguer, and K. Sen. Pmaude: Rewrite-based specification language for probabilistic object systems. Electronic Notes in Theoretical Computer Science, 153(2):213-239, 2006. 
2. R. Alur, C. Courcoubetis, N. Halbwachs, T. A. Henzinger, P. Ho, X. Nicollin, A. Olivero, J. Sifakis, and S. Yovine. The algorithmic analysis of hybrid systems. Theoretical Computer Science, 138(1):3-34, 1995.

3. R. Alur, T. Feder, and T. A. Henzinger. The benefits of relaxing punctuality. J. ACM, 43:116-146, January 1996.

4. R. Alur, S. L. Torre, and G. J. Pappas. Optimal paths in weighted timed automata. In $H S C C^{\prime} 01$, pages 49-62. Springer, 2001.

5. B. Barbot, T. Chen, T. Han, J.-P. Katoen, and A. Mereacre. Efficient ctmc model checking of linear real-time objectives. In P. A. Abdulla and K. R. M. Leino, editors, TACAS, volume 6605 of Lecture Notes in Computer Science, pages 128142. Springer, 2011.

6. G. Behrmann, A. Fehnker, T. Hune, K. G. Larsen, P. Pettersson, J. Romijn, and F. W. Vaandrager. Minimum-cost reachability for priced timed automata. In M. D. D. Benedetto and A. L. Sangiovanni-Vincentelli, editors, $H S C C$, volume 2034 of Lecture Notes in Computer Science, pages 147-161. Springer, 2001.

7. P. Bouyer, K. G. Larsen, and N. Markey. Model checking one-clock priced timed automata. Logical Methods in Computer Science, 4(2), 2008.

8. F. Cassez and K. G. Larsen. The impressive power of stopwatches. In C. Palamidessi, editor, CONCUR, volume 1877 of Lecture Notes in Computer Science, pages 138-152. Springer, 2000.

9. J.-M. Couvreur. On-the-fly verification of linear temporal logic. In FM '99, pages 253-271, 1999.

10. A. David, K. G. Larsen, A. Legay, M. Mikucionis, D. Poulsen, J. van Vliet, and Z. Wang. Statistical model checking for networks of priced timed automata. In Proceedings of Formal Modeling and Analysis of Timed Systems, Aalborg, 2011. LNCS.

11. A. David, K. G. Larsen, A. Legay, M. Mikucionis, and Z. Wang. Time for statistical model checking of real-time systems. In Proceedings of the $23^{\text {rd }}$ International Conference on Computer Aided Verification (CAV), LNCS. Springer Verlag, 2011.

12. M. Geilen. An improved on-the-fly tableau construction for a real-time temporal logic. In In International Conference on Computer Aided Verification, pages 276290. Springer, 2003.

13. M. Geilen and D. Dams. An on-the-fly tableau construction for a real-time temporal logic. In FTRTFT, pages 276-290, 2000.

14. E. M. C. Jr., O. Grumberg, and D. A. Peled. Model Checking. The MIT Press, 1999.

15. R. Koymans. Specifying real-time properties with metric temporal logic. Real-Time Syst., 2:255-299, October 1990.

16. O. Maler, D. Nickovic, and A. Pnueli. From mitl to timed automata. In FORMATS'06, pages 274-289. Springer, 2006.

17. D. Nickovic and N. Piterman. From mtl to deterministic timed automata. In 8 th International Conference on Formal Modelling and Analysis of Timed Systems, volume 6246 of $L N C S$, pages 152-167. Springer, 2010.

18. K. Sen, M. Viswanathan, and G. Agha. On statistical model checking of stochastic systems. In In Etessami, K., Rajamani, S.K., eds.: CAV. Volume 3576 of Lecture Notes in Computer Science, pages 266-280. Springer, 2005.

19. H. L. S. Younes. Verification and Planning for Stochastic Processes with Asynchronous Events. PhD thesis, Carnegie Mellon University, 2005.

20. P. Zuliani, A. Platzer, and E. M. Clarke. Bayesian statistical model checking with application to simulink/stateflow verification. In $H S C C$ '10, pages $243-252$, New York, NY, USA, 2010. ACM. 


\section{A WMTL $_{\leq}$is Not Deterministic}

In this section we show that there is no procedure that for a given $\mathrm{WMTL}_{\leq}$ formula $\varphi$ produces a deterministic MWTA $A_{\varphi}^{\text {det }}$ such, that $\mathcal{L}(\varphi)=\mathcal{L}\left(A_{\varphi}^{\text {det }}\right)$.

We demonstrate this by proving that for a formula

$$
\varphi \equiv \diamond_{\leq 1}^{\tau}\left(p \wedge \square_{\leq 1}^{\tau}(\neg r) \wedge \diamond_{\leq 1}^{\tau}(q)\right)
$$

there does not exist exact and deterministic monitor. In fact, this formula also belongs to $\mathrm{MITL}_{\leq}$, so we also prove that $\mathrm{MITL}_{\leq}$is not deterministic.

Thus, we extend the result of ${ }^{[7]}$, in which it has been shown that $\operatorname{MTLL}_{[a, b]}$ is not deterministic. To give a formal proof that $\varphi$ is not determinizable, one can adapt the proof of ${ }^{[7]}$ that $\square_{\leq 1}^{\tau}\left(p \Longrightarrow \diamond_{[1,2]}^{\tau}(\neg q)\right) \operatorname{MITL}_{[a, b]}$ formula is not determinizable. Here we will provide a more simple proof that doesn't use deep results from the theory of Timed Automata.

Let's assume, that there exists a deterministic MWTA $A_{\varphi}^{\text {det }}$ with $n$ clocks such, that $\mathcal{L}(\varphi)=\mathcal{L}\left(A_{\varphi}^{\text {det }}\right)$. Now, let's consider the set $V$ of timed words of the form $\left(p, t_{1}\right)\left(p, t_{2}\right) \ldots\left(p, t_{n+3}\right)$ that are monotonic (i.e. $\left.t_{i+1}>t_{i}\right)$, consist only of $p$ actions, have duration 1 (i.e. $t_{n+3}=1$ ) and start from zero (i.e. $t_{1}=0$ ). After reading a word from the set $V$ a value of each clock $x_{i}$ of $A_{\varphi}^{\text {det }}$ is equal to $1-t_{a_{i}}$ for some index $a_{i}$ (remind, that the clocks can be reset only when a MWTA reads a discrete action). Since $A_{\varphi}^{\text {det }}$ is deterministic and in its guards we permit only integer-valued bounds, after reading any word from $V$ the MWTA $A_{\varphi}^{\text {det }}$ will be in the same location. Moreover, the values of indexes $a_{i}$ will be the same for all words from $V$ (i.e. $A_{\varphi}^{\text {det }}$ "memorizes" the time of the events from the fixed set of numbers). Since $A_{\varphi}^{\text {det }}$ has only $n$ clocks, there exists an index $j \in[1 . . n+1]$ such that the MWTA doesn't memorize the moment when it read the discrete action $p$ number $j$. It means, that there doesn't exist index $a_{i}$ such that $a_{i}=j$ (and $x_{a_{i}}=1-t_{j}$ ).

Let's pick two timed words $u$ and $v$ from $V$ that differ only at the $j$-th position, i.e.:

$$
\begin{aligned}
& u=\left(p, t_{1}\right)\left(p, t_{2}\right) \ldots\left(p, t_{j}\right) \ldots\left(p, t_{n+3}\right) \\
& v=\left(p, t_{1}\right)\left(p, t_{2}\right) \ldots\left(p, t_{j}^{\prime}\right) \ldots\left(p, t_{n+3}\right)
\end{aligned}
$$

, and $t_{j}^{\prime}>t_{j}$.

The MWTA $A_{\varphi}^{\text {det }}$ can't distinguish $u$ and $v$, i.e. for any continuation $w$ the MWTA accepts the timed word $u \cdot w$ iff it accepts the timed word $v \cdot w$. But it can be easily seen, that this should not be the case for $w=\left(q, t_{j}+1\right)\left(r, t_{j}^{\prime}+1\right)$. Indeed, $\varphi$ is satisfied by a timed word $u^{\prime}$ and is not satisfied by a timed word $v^{\prime}$, where:

$$
\begin{aligned}
& u^{\prime}=\left(p, t_{1}\right)\left(p, t_{2}\right) \ldots\left(p, t_{j}\right) \ldots\left(p, t_{n+3}\right)\left(q, t_{j}+1\right)\left(r, t_{j}^{\prime}+1\right), \\
& v^{\prime}=\left(p, t_{1}\right)\left(p, t_{2}\right) \ldots\left(p, t_{j}^{\prime}\right) \ldots\left(p, t_{n+3}\right)\left(q, t_{j}+1\right)\left(r, t_{j}^{\prime}+1\right)
\end{aligned}
$$

\footnotetext{
${ }^{7}$ O. Maler, D. Nickovic, and A. Pnueli. Real time temporal logic: Past, present, future. In FORMATS, pages 2-16, 2005.
} 
Thus, the timed words $u^{\prime}$ and $v^{\prime}$ are distinguishable by the $\mathrm{WMTL}_{\leq}$formula $\varphi$, and are not distinguishable by its monitor $A_{\varphi}^{\text {det }}$. This violates the initial assumption, and thus $\varphi$ is not determinizable. 


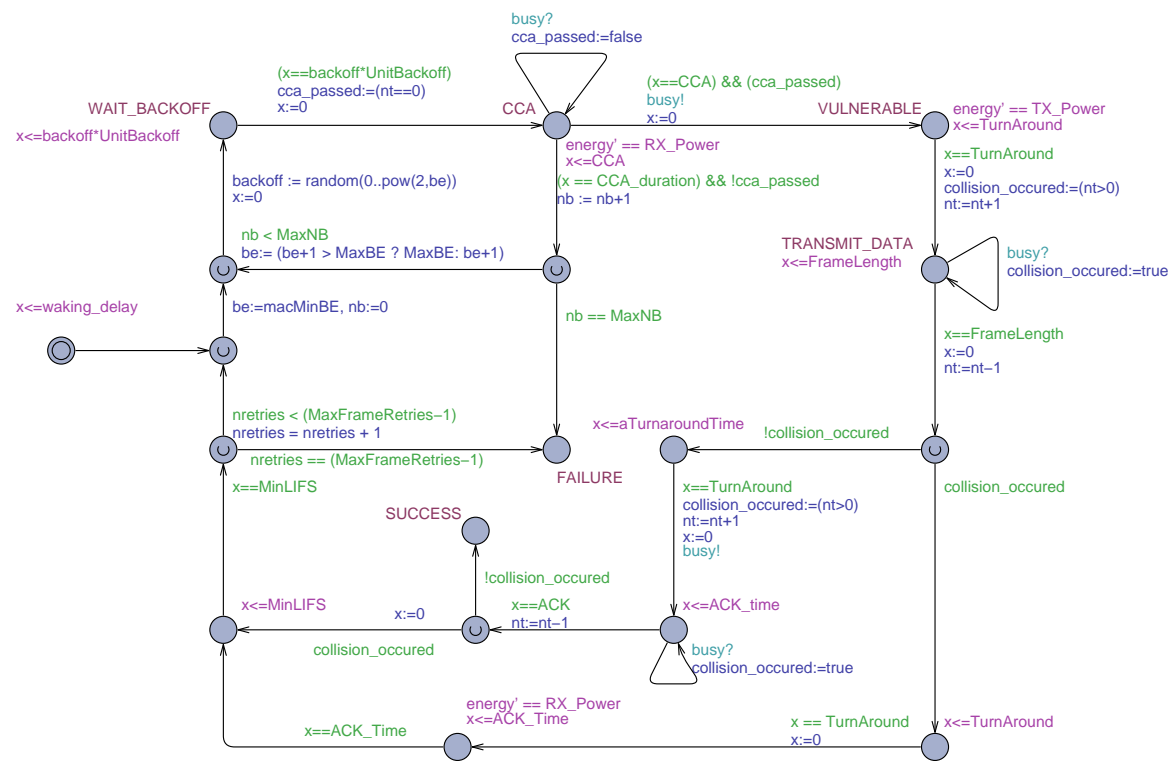

Fig. 6: Model of IEEE 802.15.4 CSMA/CA protocol

\section{B Logical Decomposition of $\mathrm{WMTL}_{\leq}$Properties}

Even though the size of monitors $A_{\varphi}^{u}$ and $A_{\varphi}^{o}$ produced by our tool chain depends exponentially on the size of $\varphi$, we observe in our experiments that in many cases complex specifications are logically composed of smaller specifications (for instance, a requirement of a complex system behavior may be a conjunction of requirements over its components). This allows us to use (smaller) monitors for logical sub-formulas of $\varphi$ instead of using one (large) monitor for $\varphi$.

We illustrate this by the following example. Consider, that $\varphi \equiv \varphi_{1} \wedge\left(\varphi_{2} \vee \varphi_{3}\right)$. Then for computing the lower bound we can construct monitors $A_{\varphi_{1}}^{u}, A_{\varphi_{2}}^{u}$ and $A_{\varphi_{3}}^{u}$ and put them all in parallel with $M$. For this parallel composition, the special clock $c^{\prime}$ grows with rate 1 iff any continuation of a trace can't satisfy $\varphi$, i.e. if the location $l_{r}^{1} \vee\left(l_{r}^{2} \wedge l_{r}^{3}\right)$ has been reached. Then it's easy to see that $\operatorname{Pr}\left[M\left\|A_{\varphi_{1}}^{u}\right\| A_{\varphi_{2}}^{u} \| A_{\varphi_{3}}^{u} \models \diamond_{\leq 1}^{c}\left(l_{a}^{1} \wedge\left(l_{a}^{2} \vee l_{a}^{3}\right)\right)\right]$ bounds the probability $\operatorname{Pr}[M \models \varphi]$ from bottom. Furthermore, we can apply similar construction for the upper bound, and thus bound the probability from both sides.

We demonstrate the applicability of this approach by the following case study.

\section{B.1 IEEE 802.15.4 CSMA/CA Protocol}

IEEE 802.15.4 standard specifies the physical layer and media access control layer for low-cost and low-rate wireless personal area networks. Devices in such networks share the same wireless medium and thus they can possibly corrupt the transmission of each other by sending data at the same time. We applied our tool 
to the analysis of Multiple Access/Collision Avoidance (CSMA/CA) network contention protocol that is used in IEEE 802.15.4 to minimize the number of collisions.

Our model of IEEE 802.15.4 CSMA/CA protocol is given at Fig. 6 .

Our goal was to estimate the probability that all the nodes can recover from a collision within a given time bound. This requirement can be specified by a $\mathrm{WMTL}_{\leq}$formula $\wedge_{i=1 . . N} \varphi_{i}$, where $N$ is a number of network nodes and $\varphi_{i}$ specifies the behavior of a single node:

$$
\varphi_{i} \equiv \square_{\leq 10000}^{\tau}\left(\text { collision }_{i} \Longrightarrow \diamond_{\leq 4000}^{\tau} \text { send }_{i}\right)
$$

We applied our tool chain to check this property for different values of $N$. CASAAL tool produced an exact monitoring automaton $A_{\varphi}^{u}$ for this formula.

\begin{tabular}{lcccc}
\hline Number of nodes & 2 & 3 & 4 & 5 \\
\hline Time to produce $A_{\varphi}^{u}$ & $<1 \mathrm{~s}$ & $3 \mathrm{~s}$ & $57 \mathrm{~s}$ & $20 \mathrm{~m} 2 \mathrm{~s}$ \\
Number of locations in $A_{\varphi}^{u}$ & 18 & 66 & 258 & 1026 \\
Number of transitions in $A_{\varphi}^{u}$ & 230 & 2049 & 16306 & 123800 \\
Time to perform SMC & $16 \mathrm{~s}$ & $1 \mathrm{~m} 1 \mathrm{~s}$ & $3 \mathrm{~m} 36 \mathrm{~s}$ & $12 \mathrm{~m} 4 \mathrm{~s}$ \\
\hline
\end{tabular}

(a) Without logical decomposition of the formula

\begin{tabular}{lccccccc}
\hline Number of nodes & 2 & 3 & 4 & 5 & 6 & 7 & 8 \\
\hline Time to produce $A_{\varphi_{1}}^{u}\|\ldots\| A_{\varphi_{N}}^{u}$ & $<1 \mathrm{~s}$ & $<1 \mathrm{~s}$ & $<1 \mathrm{~s}$ & $1 \mathrm{~s}$ & $1 \mathrm{~s}$ & $1 \mathrm{~s}$ & $2 \mathrm{~s}$ \\
Time to perform SMC & $17 \mathrm{~s}$ & $43 \mathrm{~s}$ & $1 \mathrm{~m} 24 \mathrm{~s}$ & $2 \mathrm{~m} 28 \mathrm{~s}$ & $4 \mathrm{~m} 4 \mathrm{~s}$ & $6 \mathrm{~m} 8 \mathrm{~s}$ & $9 \mathrm{~m} 52 \mathrm{~s}$ \\
SMC result & 0.96 & 0.85 & 0.66 & 0.45 & 0.24 & 0.10 & 0.05 \\
\hline
\end{tabular}

(b) With logical decomposition of the formula

Table 3: Results for IEEE CSMA/CA 802.15.4 protocol

Unfortunately, the size of $\varphi$ grows linearly with $N$, resulting in the exponential growth of the size of a monitor $A_{\varphi}^{u}$ (and the time required to build it). This slows down the overall tool chain, that is reflected on Fig. 3a (for this case study we use $\varepsilon=0.01$ and $\alpha=0.95$ statistical parameters). However, our formula decomposition technique described below helped to overcome this problem. Indeed, $\varphi$ is a conjunction of simple formulas $\varphi_{i}$, such that they and their monitors are constant in their size. This is illustrated by Fig. 3b that provides the results for the case when we use logical decomposition. 


\section{Additional Experimental Results}

\begin{tabular}{|c|r|r|r|r|}
\hline formula & automaton & states & trans & time $(\mathrm{s})$ \\
\hline \multirow{3}{*}{$\neg \diamond_{\leq 5}^{\tau} p$} & nondet & 3 & 4 & 0.02 \\
\cline { 2 - 5 } & under & 3 & 4 & 0.00 \\
\cline { 2 - 5 } & over & 3 & 4 & 0.02 \\
\cline { 2 - 5 } & Geilen & 4 & 6 & \\
\hline \hline \multirow{3}{*}{$\square_{\leq 100}^{\tau} \diamond_{\leq 5}^{\tau} p$} & nondet & 5 & 12 & 0.02 \\
\cline { 2 - 5 } & under & 5 & 12 & 0.01 \\
\cline { 2 - 5 } & over & 5 & 12 & 0.02 \\
\cline { 2 - 5 } & Geilen & 10 & 22 & \\
\hline \hline \multirow{3}{*}{$\diamond_{\leq 5}^{\tau}\left(\square_{\leq 1}^{\tau} p \vee \square_{\leq 1}^{\tau} q\right)$} & nondet & 5 & 11 & 0.02 \\
\cline { 2 - 5 } & under & 9 & 37 & 0.02 \\
\cline { 2 - 5 } & over & 9 & 37 & 0.02 \\
\cline { 2 - 5 }$p \rightarrow\left(\square_{\leq 5}^{\tau}\left(q \rightarrow \square_{\leq 1}^{\tau} r\right)\right)$ & Geilen & 11 & 21 & \\
\cline { 2 - 5 } & nondet & 5 & 14 & 0.02 \\
\cline { 2 - 5 } & under & 5 & 14 & 0.01 \\
\cline { 2 - 5 } & Geilen & 15 & 48 & 0.01 \\
\hline \hline
\end{tabular}

Table 4: Additional experimental results for $\mathrm{WMTL}_{\leq}$formulas for Section 4. 


\section{Proof of Theorem 1}

In this Appendix we provide a detailed proof for our main Theorem 1. The following two Lemma's proves the validity of the distribution laws of Lemma 1.

Lemma 2. Let $\omega$ be an extended weighted word.

1. If $\omega \models_{e} g \vee\left(f \wedge O\left((x \leq d) \wedge\left(f \mathrm{U}_{\leq d-x}^{c} g\right)\right)\right)$, then $\omega \models_{e} f \mathrm{U}_{\leq d}^{c} g$.

2. If $\omega \models_{e} g \vee\left(f \wedge \operatorname{unch}(x) \wedge O\left((x \leq d) \wedge\left(f \mathrm{U}_{\leq d-x}^{c} g\right)\right)\right)$, then $\omega \models_{e} f \mathrm{U}_{\leq d-x}^{c} g$.

3. If $\omega=_{e} g \wedge\left(f \vee\left(r s t(x) \wedge O\left(\left((x \leq d) \wedge\left(f \mathrm{R}_{\leq d-x}^{c} g\right)\right) \vee(x>d)\right)\right)\right)$, then $\omega=_{e}$ $f \mathrm{R}_{\leq d}^{c} g$.

4. If $\omega \models_{e} g \wedge\left(f \vee O\left(\left((x \leq d) \wedge\left(f \mathrm{R}_{\leq d-x}^{c} g\right)\right) \vee(x>d)\right)\right)$, then $\omega \models_{e} f \mathrm{R}_{\leq d-x}^{c} g$.

\section{Proof}

1. Assume that $\omega \models_{e} g \vee\left(f \wedge O\left((x \leq d) \wedge\left(f \mathrm{U}_{\leq d-x}^{c} g\right)\right)\right)$.

If $\omega \models_{e} g$, then the conclusion is true.

If $\omega \not \nvdash_{e} g$, then $\omega \models_{e} f \wedge O\left((x \leq d) \wedge\left(f \mathrm{U}_{<d-x}^{c} g\right)\right)$.

Hence $\omega \models_{e} f$, and $\omega^{1} \models_{e}(x \leq d) \wedge\left(f \mathrm{U}_{\leq d-x}^{c} g\right)$.

So $\omega \models_{e} f \mathrm{U}_{\leq d}^{c} g$.

2., 3., 4. Similar.

Definition 5. Given a weighted word $w=\left(a_{0}, v_{0}\right)\left(a_{1}, v_{1}\right)\left(a_{2}, v_{2}\right) \ldots$, and a clock valuation $\nu_{0}$, an extended weighted word $\bar{w}=\left(a_{0}, v_{0}, \nu_{0}\right)\left(a_{1}, v_{1}, \nu_{1}\right)\left(a_{2}, v_{2}, \nu_{2}\right) \ldots$ can be defined as follows.

1. If $x \in X_{\varphi}$ is a local clock assigned to $f \mathrm{U}_{\leq d}^{c}$, then $\bar{w}^{i} \models$ unch $(x)$ iff $\bar{w}^{i} \models_{e}$ $f \mathbf{U}_{\leq d-x}^{c} g$, and $w^{i} \not \models g$.

2. If $x \in X_{\varphi}$ is a local clock assigned to $f \mathrm{R}_{\leq d}^{c}$ g, then $\bar{w}^{i} \models \operatorname{rst}(x)$ iff $\bar{w}^{i} \models_{e}$ $f \mathrm{R}_{\leq d}^{c} g$, and $w^{i} \not \models f$.

Lemma 3. Let $w$ be a weighted word, and $\bar{w}$ be an extended weighted word defined in Definition 5, then

1. If $\bar{w}^{i} \models_{e} f \mathrm{U}_{\leq d}^{c} g$, then $\bar{w}^{i} \models_{e} g \vee\left(f \wedge O\left((x \leq d) \wedge\left(f \mathrm{U}_{\leq d-x}^{c} g\right)\right)\right)$.

2. If $\bar{w}^{i} \models_{e} f \mathrm{U}_{\leq d-x}^{\bar{c}} g$, then $\bar{w}^{i} \models_{e} g \vee\left(f \wedge\right.$ unch $\left.(x) \wedge O\left((x \leq d) \wedge\left(f \mathrm{U}_{\leq d-x}^{c} g\right)\right)\right)$

3. If $\bar{w}^{i} \models_{e} f \mathrm{R}_{\leq d}^{c} g$, then $\bar{w}^{i} \models_{e} g \wedge\left(f \vee\left(r s t(x) \wedge O\left(\left((x \leq d) \wedge\left(f \mathrm{R}_{\leq d-x}^{c} g\right)\right) \vee(x>\right.\right.\right.$ d)) ))

4. If $\bar{w}^{i} \models_{e} f \mathrm{R}_{\leq d-x}^{c} g$, then $\bar{w}^{i} \models_{e} g \wedge\left(f \vee O\left(\left((x \leq d) \wedge\left(f \mathrm{R}_{\leq d-x}^{c} g\right)\right) \vee(x>d)\right)\right)$

\section{Proof}

1. Assume that $\bar{w}^{i} \models_{e} f \mathrm{U}_{\leq d}^{c} g$.

(a) If $\bar{w}^{i} \models_{e} g$, the conclusion is true.

(b) If $\bar{w}^{i} \not \nvdash_{e} g$ and $\bar{w}^{i} \models_{e} f \mathrm{U}_{\leq d-x}^{c} g$,

then by Definition $5, \bar{w}^{i} \models \operatorname{unch}(x)$,

From $\bar{w}^{i} \models_{e} f \mathrm{U}_{\leq d-x}^{c} g$, we have $\bar{w}^{i+1} \models_{e}(x \leq d) \wedge\left(f \mathrm{U}_{\leq d-x}^{c} g\right)$.

Thus $\bar{w}^{i} \models_{e} f \wedge O\left((x \leq d) \wedge\left(f \mathrm{U}_{\leq d-x}^{c} g\right)\right)$. 
(c) If $\bar{w}^{i} \not \nvdash_{e} g$ and $\bar{w}^{i} \not \nvdash_{e} f \mathrm{U}_{\leq d-x}^{c} g$,

then $\bar{w}^{i} \not \nvdash_{e}$ unch $(x)$, and $x$ will be reset at $i$.

From $\bar{w}^{i} \models_{e} f \mathrm{U}_{\leq d}^{c} g$, we know that $\bar{w}^{i+1} \models_{e}(x \leq d) \wedge\left(f \mathrm{U}_{\leq d-x}^{c} g\right)$ and $\bar{w}^{i} \models_{e} f$.

So $\bar{w}^{i} \models_{e} f \wedge O\left((x \leq d) \wedge\left(f \mathrm{U}_{\leq d-x}^{c} g\right)\right)$.

2. Assume that $\bar{w}^{i} \models_{e} f \mathrm{U}_{\leq d-x}^{c} g$.

(a) If $\bar{w}^{i} \models_{e} g$, the conclusion is true.

(b) If $\bar{w}^{i} \not \nvdash_{e} g$, then by Definition $5, \bar{w}^{i} \models u n c h(x)$,

From $\bar{w}^{i} \models_{e} f \cup_{\leq d-x}^{c} g$, we know that $\bar{w}^{i} \models_{e} f$ and $\bar{w}^{i+1} \models_{e}(x \leq$ $d) \wedge\left(f \mathrm{U}_{\leq d-x}^{c} g\right)$.

So $\bar{w}^{i} \models_{e} f \wedge \operatorname{unch}(x) \wedge O\left((x \leq d) \wedge\left(f \cup_{\leq d-x}^{c} g\right)\right)$.

3. Assume that $\bar{w}^{i} \models_{e} f \mathrm{R}_{\leq d}^{c} g$.

(a) If $\bar{w}^{i} \models_{e} f$, then $\bar{w}^{i} \models_{e} f \wedge g$, and the conclusion is true.

(b) If $\bar{w}^{i} \not \nvdash_{e} f$, then by Definition $5, \bar{w}^{i} \models \operatorname{rst}(x)$.

From $\bar{w}^{i} \models_{e} f \mathrm{R}_{\leq d}^{c} g$, we know that $\bar{w}^{i+1} \models_{e}\left((x \leq d) \wedge\left(f \mathrm{R}_{\leq d-x}^{c} g\right)\right) \vee(x>$ d).

Thus we get the conclusion that $\bar{w}^{i} \models_{e} g \wedge r s t(x) \wedge O(((x \leq d) \wedge$ $\left.\left.\left(f \mathrm{R}_{\leq d-x}^{c} g\right)\right) \vee(x>d)\right)$.

4. Assume that $\bar{w}^{i} \models_{e} f \mathrm{R}_{\leq d-x}^{c} g$.

(a) If $\bar{w}^{i} \models_{e} f$, then $\bar{w}^{i} \models_{e} f \wedge g$, and the conclusion is true.

(b) If $\bar{w}^{i} \not \nvdash_{e} f$ and $\bar{w}^{i} \models_{e} f \mathrm{R}_{\leq d}^{c} g$,

then by Definition $5, \bar{w}^{i} \models \operatorname{rst}(x)$,

From $\bar{w}^{i} \models_{e} f \mathrm{R}_{\leq d}^{c} g$, we know that $\bar{w}^{i+1} \models_{e}\left((x \leq d) \wedge\left(f \mathrm{R}_{\leq d-x}^{c} g\right)\right) \vee(x>$ d).

Thus $\bar{w}^{i} \models_{e} g \wedge O\left(\left((x \leq d) \wedge\left(f \mathrm{R}_{<d-x}^{c} g\right)\right) \vee(x>d)\right)$.

(c) If $\bar{w}^{i} \not \nvdash_{e} f$ and $\bar{w}^{i} \not \nvdash_{e} f \mathrm{R}_{\leq d}^{c} g$, then $\bar{w}^{i} \not \nvdash_{e} \operatorname{rst}(x)$.

By $\bar{w}^{i} \models_{e} f \mathrm{R}_{\leq d-x}^{c} g$, we get that $\bar{w}^{i+1} \models_{e}\left((x \leq d) \wedge\left(f \mathrm{R}_{\leq d-x}^{c} g\right)\right) \vee(x>d)$.

Thus $\bar{w}^{i} \models_{e} g \wedge O\left(\left((x \leq d) \wedge\left(f \mathrm{R}_{\leq d-x}^{c} g\right)\right) \vee(x>d)\right)$.

Using the equivalences of Lemma 1 any formula of $\mathrm{MITL}_{\leq}$may be transformed into a disjunctive normal formal containing several basic conjunctions, as stated and proved by the following Lemma.

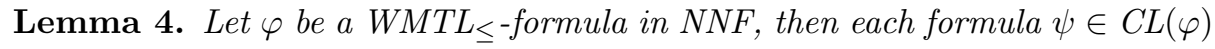
can be translated into a disjunction of basic conjunctions:

$$
\bigvee_{j=0}^{k}\left(\alpha_{j} \wedge g_{j} \wedge \operatorname{rst}\left(X_{j}\right) \wedge \operatorname{unch}\left(Y_{j}\right) \wedge O\left(\psi_{j}\right)\right)
$$

and

1. For every extended weighted word $w$, if $w \models_{e} \alpha_{j} \wedge g_{j} \wedge \operatorname{rst}\left(X_{j}\right) \wedge \operatorname{unch}\left(Y_{j}\right) \wedge$ $O\left(\psi_{j}\right)$ for some $j$, then $w \models_{e} \psi$. 
2. For every weighted word $w$, if $\bar{w} \models_{e} \psi$, then there exists $j$ such that $\bar{w} \models_{e}$ $\alpha_{j} \wedge g_{j} \wedge \operatorname{rst}\left(X_{j}\right) \wedge \operatorname{unch}\left(Y_{j}\right) \wedge O\left(\psi_{j}\right)$.

Proof

1. Define Length $(\psi)$ for $\psi \in C L(\varphi)$ as follows.

(a) Length $(p)=\operatorname{Length}(\neg p)=\operatorname{Length}(x \leq d)=\operatorname{Length}(x>d)=\operatorname{Length}(O \phi)=1$;

(b) Length $\left(\phi_{1} \vee \phi_{2}\right)=\operatorname{Length}\left(\phi_{1} \wedge \phi_{2}\right)=\operatorname{Length}\left(\phi_{1} \mathrm{U}_{\leq d}^{c} \phi_{2}\right)$

$=\operatorname{Length}\left(\phi_{1} \mathrm{R}_{\leq d}^{c} \phi_{2}\right)=\operatorname{Length}\left(\phi_{1} \mathrm{U}_{\leq d-x}^{c} \phi_{2}\right)=\operatorname{Length}\left(\phi_{1} \mathrm{R}_{\leq d-x}^{c} \phi_{2}\right)$ $=\operatorname{Length}\left(\phi_{1}\right)+\operatorname{Length}\left(\phi_{2}\right)+1$.

2. By induction on $\operatorname{Length}(\psi)$ for $\psi \in C L(\varphi)$.

Now let us recall the Main Theorem 1.

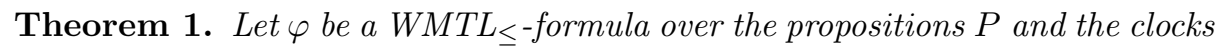
$C$ and is in NNF. Let the $M W T A A_{\varphi}=\left(L, \ell_{0}, \ell_{a}, C_{M}, E, m\right)$ over the clocks $C$ and the actions $\mathcal{A}=2^{P}$ be defined as follows:

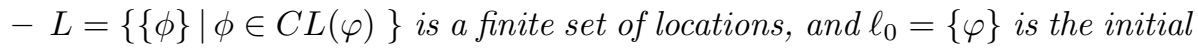
location;

$-\ell_{a}=\{$ true $\}$ is the accepting location;

$-C_{M}=X_{\varphi}$ is the set of all local clocks for $\varphi$;

$-\left(\left\{f_{1}\right\}, a, g, \lambda,\left\{f_{2}\right\}\right) \in E$ iff $\alpha \wedge g \wedge \operatorname{rst}(X) \wedge \operatorname{unch}(Y) \wedge O\left(f_{2}\right)$ is a basic conjunction of $f_{1}$ and that a satisfies $\alpha$, and for each $x \in X_{\varphi}$ of $\mathrm{U}_{\leq- \text {-type, } x} \in \lambda$ iff $x \notin Y$, and for each $x \in X_{\varphi}$ of $\mathrm{R}_{\leq}$-type, $x \in \lambda$ iff $x \in X$;

$-m$ is defined by $m\left(x_{\phi_{1} \cup_{\leq d}^{c} \phi_{2}}\right)=c$ and $m\left(x_{\phi_{1} R_{\leq d}^{c} \phi_{2}}\right)=c$.

Then $L(\varphi)=L\left(A_{\varphi}\right)$.

Proof

$L\left(A_{\varphi}\right) \subseteq L(\varphi)$. Let $w=\left(a_{0}, v_{0}\right)\left(a_{1}, v_{1}\right)\left(a_{2}, v_{2}\right) \ldots$ be a weighted word in $\mathcal{L}\left(A_{\varphi}\right)$, then there are $\psi_{0}, \psi_{1}, \psi_{2}, \ldots, \psi_{n} \in C L(\varphi)$ and clock valuations

$\nu_{0}, \nu_{1}, \nu_{2}, \ldots, \nu_{n}$ such that $\psi_{0}=\varphi, \psi_{n}=$ true, $\psi_{i} \stackrel{a_{i}, g_{i}, r_{i}}{\longrightarrow} \psi_{i+1}$ is a transition of $A_{\varphi}$, and $\alpha_{i} \wedge g_{i} \wedge \operatorname{rst}\left(X_{i}\right) \wedge \operatorname{unch}\left(Y_{i}\right) \wedge O\left(\psi_{i+1}\right)$ is a basic conjunction of $\psi_{i}, a_{i} \models \alpha_{i}, \nu_{i} \models g_{i}$ and for each $x \in X_{\varphi}$ : if $x \in r_{i}$ then $\nu_{i+1}=v_{i+1}(m(x))-$ $v_{i}(m(x))$ else $\nu_{i+1}=\nu_{i}+v_{i+1}(m(x))-v_{i}(m(x))$.

Then we get an extended weighted word $\omega=\left(a_{0}, v_{0}, \nu_{0}\right)\left(a_{1}, v_{1}, \nu_{1}\right)\left(a_{2}, v_{2}, \nu_{2}\right) \ldots$

Now we prove by induction in $n-i$, that for all $i \leq n$ : $\omega^{i}=\psi_{i}$.

(a) If $i=n$, then $\psi_{n}=$ true and $\omega^{n} \models \psi_{n}$.

(b) Assume $\omega^{i} \models \psi_{i}$ is true for all $i>=k+1$, now we show that $\omega^{k} \models \psi_{k}$ is true.

From $\psi_{k} \stackrel{a_{k}, g_{k}, r_{k}}{\longrightarrow} \psi_{k+1}$, we know that $\omega^{k} \models \alpha_{k} \wedge g_{k} \wedge r \operatorname{st}\left(X_{k}\right) \wedge u n c h\left(Y_{k}\right) \wedge$ $O\left(\psi_{k+1}\right)$, so from Lemma $4, \omega^{k} \models \psi_{k}$. 
$L(\varphi) \subseteq L\left(A_{\varphi}\right)$. Let $w=\left(a_{0}, v_{0}\right)\left(a_{1}, v_{1}\right)\left(a_{2}, v_{2}\right) \ldots$ be a weighted word in $\mathcal{L}(\varphi)$, and $\nu_{0}$ is an initial valuation for clocks in $X_{\varphi}$. Let

$\bar{w}=\left(a_{0}, v_{0}, \nu_{0}\right)\left(a_{1}, v_{1}, \nu_{1}\right)\left(a_{2}, v_{2}, \nu_{2}\right) \ldots$ be the extended weighted word defined in Definition 5.

From $w \in \mathcal{L}(\varphi)$, we know that $\bar{w}=\varphi$.

By Lemma 4 , there is a basic conjunction $\alpha_{0} \wedge g_{0} \wedge r s t\left(X_{0}\right) \wedge u n c h\left(Y_{0}\right) \wedge O\left(\varphi_{1}\right)$ of $\varphi$ such that $\bar{w}=\alpha_{0} \wedge g_{0} \wedge r s t\left(X_{0}\right) \wedge u n c h\left(Y_{0}\right) \wedge O\left(\varphi_{1}\right)$.

Then $\varphi \stackrel{a_{0}, g_{0}, r_{0}}{\longrightarrow} \varphi_{1}$ is a transition of $A_{\varphi}, \bar{w}^{1} \models \varphi_{1}$, and for all $x \in X_{\varphi}$ : if $x \in r_{0}$ then $\nu_{1}=v_{1}(m(x))-v_{0}(m(x))$ else $\nu_{1}=\nu_{0}+v_{1}(m(x))-v_{0}(m(x))$, where $r_{0}$ the reset set of local clocks defined by $\bar{w}$ at position 0 ( if $\bar{w}=_{e}$ $r s t(x)$ and $x$ is local clock of $\mathrm{R}_{\leq}$-type, then $x \in r_{0}$; if $\bar{w} \not \nvdash_{e}$ unch $(x)$ and $x$ is local clock of $\mathrm{U}_{\leq}$-type, then $x \in r_{0}$; otherwise $\left.x \notin r_{0}\right)$.

Similarly, we can get a sequence $\varphi_{2}, \varphi_{3}, \varphi_{4}, \ldots$ of formulas from $C L(\varphi)$ such that $\varphi_{i} \stackrel{a_{i}, g_{i}, r_{i}}{\longrightarrow} \varphi_{i+1}$ is a transition of $A_{\varphi}$ and $\bar{w}_{i} \models \alpha_{i} \wedge g_{i} \wedge \operatorname{rst}\left(X_{i}\right) \wedge$ $\operatorname{unch}\left(Y_{i}\right) \wedge O\left(\varphi_{i+1}\right)$ for all $i \in \mathbb{N}$.

Now if we can prove that some $\varphi_{i}$ is in the accepting location, then $w$ will be accepted by $A_{\varphi}$.

To do this, we define the depth $\operatorname{dep}(\phi)$ for formulas in $\operatorname{CL}(\varphi)$.

(a) $\operatorname{dep}(p)=\operatorname{dep}(\neg p)=\operatorname{dep}(x \leq d)=\operatorname{dep}(x>d)=0$;

(b) $\operatorname{dep}\left(\phi_{1} \vee \phi_{2}\right)=\operatorname{dep}\left(\phi_{1} \wedge \phi_{2}\right)=\max \left\{\operatorname{dep}\left(\phi_{1}\right), \operatorname{dep}\left(\phi_{2}\right)\right\}$;

(c) $\operatorname{dep}(O \phi)=\operatorname{dep}\left(\phi_{1}\right)+1$;

(d) $\operatorname{dep}\left(\phi_{1} \mathrm{U}_{\leq d}^{c} \phi_{2}\right)=\operatorname{dep}\left(\phi_{1} \mathrm{R}_{\leq d}^{c} \phi_{2}\right)=\max \left\{\operatorname{dep}\left(\phi_{1}\right), \operatorname{dep}\left(\phi_{2}\right)\right\}+2$;

(e) $\operatorname{dep}\left(\phi_{1} \mathrm{U}_{\leq d-x}^{c} \phi_{2}\right)=\operatorname{dep}\left(\phi_{1} \mathrm{R}_{\leq d-x}^{c} \phi_{2}\right)=\max \left\{\operatorname{dep}\left(\phi_{1}\right), \operatorname{dep}\left(\phi_{2}\right)\right\}+1$.

Then $\operatorname{dep}(\varphi) \geq \operatorname{dep}\left(\varphi_{1}\right) \geq \operatorname{dep}\left(\varphi_{2}\right) \geq \operatorname{dep}\left(\varphi_{3}\right) \geq \ldots$ and there exists $N$ such that for all $i>=N$ : $\operatorname{dep}\left(\varphi_{i}\right)=\operatorname{dep}\left(\varphi_{N}\right)$.

If $\operatorname{dep}\left(\varphi_{N}\right)>0$, then some $\phi_{1} \mathrm{U}_{\leq d-x}^{c} \phi_{2}$ or $\phi_{1} \mathrm{R}_{\leq d-x}^{c} \phi_{2}$ will remain in $\varphi_{i}$ for all $i>=N$, and $x$ will not be reset and will not exceed $d$ for all $i>N$. This is not possible, because all infinite weighted words are assumed to be cost-diverging.

Thus $\operatorname{dep}\left(\varphi_{N}\right)$ must be zero, and $\varphi_{N+1}$ will be in the accepting location. So $w \in \mathcal{L}\left(A_{\varphi}\right)$. 